UVX 2008 (2009) 35-43

(C) EDP Sciences, 2009

DOI: $10.1051 / \mathrm{uvx} / 2009007$

\title{
Electron-ion vector correlations for the study of photoionization of molecules in the UVX range: From synchrotron radiation to short light pulses
}

\author{
C. Elkharrat ${ }^{1,2}$, Y.J. Picard ${ }^{1,2}$, P. Billaud ${ }^{1,2}$, J.C. Houver ${ }^{1,2}$ and D. Dowek ${ }^{1,2}$ \\ 1 Université Paris-Sud 11, UMR 8625, Laboratoire des Collisions Atomiques et Moléculaires, \\ Bât. 351, Université Paris-Sud, 91405 Orsay, France \\ 2 CNRS, UMR 8625, Laboratoire des Collisions Atomiques et Moléculaires, Bât. 351, \\ Université Paris-Sud, 91405 Orsay, France
}

\begin{abstract}
Molecular frame photoemission is a very sensitive probe of the photoionization (PI) dynamics of molecules. The electron-ion vector correlation (VC) method takes advantage of dissociative photoionization (DPI) reactions of small molecules to measure such observables. It relies on the coincident detection of the photoelectron and the ion fragment emitted from the same DPI event and the determination of their velocity vectors. Most of the VC studies so far have been performed using synchrotron radiation (SR) light sources which benefit from a high repetition rate $(1-10 \mathrm{MHz})$ favorable for coincidence experiments. In this paper we discuss the extension of this method to the study of PI processes induced by ultra-short VUV light sources, which provide the capability for investigating processes characterized by femtosecond or subfemtosecond dynamics. We first illustrate the $\mathrm{VC}$ method by the report of recent results of a comparative study of resonant photoionization of the $\mathrm{H}_{2}$ and $\mathrm{D}_{2}$ molecules induced by VUV circularly polarized synchrotron radiation at SOLEIL in the region involving resonant excitation of $\mathrm{Q}_{1}$ and $\mathrm{Q}_{2}$ doubly excited state series. This problem is of particular interest since autoionization and dissociation of selected $\mathrm{Q}_{1}$ or $\mathrm{Q}_{2}$ states occur on a comparable time scale of few femtoseconds, which implies a coupling between the electronic and nuclear motion. The extension of the VC method using femtosecond laser sources is then demonstrated by results for multiphoton PI of the Xe atom induced by $70 \mathrm{fs}$ pulses centered at $400 \mathrm{~nm}$ delivered by the SOFOCKLE and PLFA sources (SLIC, CEA-Saclay) after frequency doubling of a $1 \mathrm{kHz}$ Ti:sapphire laser.
\end{abstract}

\section{INTRODUCTION}

Interaction of UV or X rays with simple molecular systems creates the conditions for the investigation of numerous interesting problems in chemical physics. Considering first one-photon single ionization of molecules, photoionization results from the coupling induced by the light field between the initial neutral state of the molecule and the final state composed of the ionic state and the electron scattered in the continuum. In weak field conditions, this interaction is completely described by the complex dipole matrix elements which write: $I_{l m \mu}^{M_{i} M_{f}}=\left\langle\psi_{M_{i}}^{i}\left|d_{\mu}\right| \phi_{M_{f}}^{f} \psi_{i m}^{(-)}\right\rangle$for linear molecules, where $M_{i}$ and $M_{f}$ are the total orbital angular momentum of the initial and final states about the molecular axis, $m$ and $\mu$ the corresponding projections of the momentum of the electron for the $l m$ partial wave of the scattering wave function and the photon, respectively [1,2]. Through the determination of the dipole matrix elements, the study of photoionization of molecules enables a detailed description of the static electronic correlation in the bound initial (neutral) and final (ionic) states, as well as the dynamic correlation induced between the scattered electron and the electrons of the target $[3,4]$. It is also a mean to probe the structure of the ionization continuum of molecules in terms of resonances: shape resonances and autoionizing states, which may lead to interesting interference schemes due to the coherent superposition of resonant and non-resonant (direct ionization) PI reactions [5,6]. Measuring molecular frame photoemission from fixed-in-space molecules, aligned or oriented with respect to the light polarization vector, provides the most complete access to the dipole matrix elements for a photoionization reaction, their magnitude and relative phases $[3,4,7,8]$. 
If one considers photoionization of molecules in the electronic ground state, there are formally two ways to proceed. The use of short intense laser pulses [9] to spatially align molecules, which can be ionized after a controlled delay in field free conditions with a XUV femtosecond pulse [10], has opened recently a possibility to measure molecular frame photoemission, particularly valuable for the study of non-dissociative PI reactions under the condition of a high degree of initial alignment. The alternative approach, presented in this paper, is to take advantage of dissociative photoionization (DPI) reactions to measure the molecular frame photoelectron angular distributions (MFPADs) from an assembly of isotropically oriented molecules, using the vector correlation $(\mathrm{VC})$ method $[11,12]$ or another momentum spectroscopy like the COLTRIMS method [13]. Indeed, for linear molecules breaking into two fragments in the axial recoil approximation [14], i.e. when dissociation is much faster than the period of molecular rotation (or bending for polyatomic molecules), the MFPADs can be determined unambiguously by recording the velocity vectors of the ion fragment, $\mathbf{V}_{\mathbf{A}+}$, which is a signature of the molecular axis orientation at the instant of PI, and that of the photoelectron, $\mathbf{V}_{\mathbf{e}}$, produced from the same PI event. This has been illustrated in the investigation of single ionization processes corresponding to valence or inner-valence ionization induced by absorption of a VUV photon [8,14-17]:

$$
\mathrm{ABC}+h v(\hat{\mathbf{e}}) \rightarrow \mathrm{ABC}^{+*}+\mathrm{e} \rightarrow \mathrm{A}^{+}+\mathrm{BC}^{*}+\mathrm{e}
$$

The $\left(\mathbf{V}_{\mathbf{A}+}, \mathbf{V}_{\mathbf{e}}, \hat{\mathbf{e}}\right)$ correlation leads to the MFPAD which is a function of three angles $I\left(\chi, \theta_{e}, \phi_{e}\right)$, where $\chi$ is the polar angle which characterizes the orientation of the molecule with respect to the polarization axis $\hat{\mathbf{e}}$, and $\left(\theta_{e}, \phi_{e}\right)$ characterize the emission direction of the photoelectron in the molecular frame [18]. Considering a non-linear molecule, the MFPAD is a function of increasing complexity: valence shell PI producing generally a single charged fragment cannot give access to the complete MFPAD. The VC method provides then the recoil frame photoelectron angular distribution (RFPAD) which implies an average of the original MFPAD over some of the internal coordinates of the molecular object leading to a more limited information in terms of dipole matrix elements. Comparing the measured RFPADs with the computed MFPADs can nevertheless be used to determine the predissociation time or the bending angle of the molecular ionic state produced by PI [19].

Studying the interaction of molecules with $\mathrm{X}$ ray photons, which causes inner-shell ionization, opens additional problematics and possibilities. $\mathrm{K}$ shell ionization of molecules involving relatively light atoms $\left(\mathrm{NO}, \mathrm{CO}, \mathrm{N}_{2} \mathrm{O} \ldots\right.$. [20-23] similar to Eq. (1) produces a molecular ion $\mathrm{ABC}^{+*}$ which possesses an inner-shell vacancy, and most frequently relaxes by Auger decay. Dissociative ionization then results in the production of two or more ionic fragments. The VC method extended to the detection of all ionic fragments, the photoelectron, and the determination of their velocities $\left(\mathbf{V}_{\mathbf{A}+}, \mathbf{V}_{\mathbf{B}+}, \ldots \mathbf{V}_{\mathbf{e}}, \hat{\mathbf{e}}\right)$ provides perspectives both for the experimental determination of the MFPAD [24] and for the investigation of the nuclear dynamics which may occur during and after the Auger decay.

The paper is divided in two parts. After a brief description of the experimental method, we report selected results of a comparative study of PI of the $\mathrm{H}_{2}$ and $\mathrm{D}_{2}$ molecules by VUV synchrotron radiation circularly polarized light corresponding to the resonant excitation of $\mathrm{Q}_{1}\left(2 \mathrm{p} \sigma_{\mathrm{u}}, \mathrm{nl} \lambda\right)$ and $\mathrm{Q}_{2}\left(2 \mathrm{p} \pi_{\mathrm{u}}, \mathrm{nl} \lambda\right)$ doubly excited state series. We note that (i) MFPADs for linearly polarized light perpendicular to the molecular axis have been reported for resonant PI of $\mathrm{D}_{2}$ at $33.25 \mathrm{eV}$ photon energy and compared with four body calculations [25] (ii) we have previously reported MFPADs for any orientation of the $\mathrm{H}_{2}$ molecule with respect to linearly polarized light at $h v=20,28$ and $32.5 \mathrm{eV}$ [17]. Finally we have reported preliminary results for resonant $\mathrm{PI}$ of $\mathrm{D}_{2}$ at $32.5 \mathrm{eV}$ induced by circularly polarized light [26] at Super ACO compared with the four body calculations of F. Martin et al. [25]. Throughout the paper, we refer to a representation of the $\mathrm{H}_{2}\left(\mathrm{D}_{2}\right)$ and $\mathrm{H}_{2}^{+}\left(\mathrm{D}_{2}^{+}\right)$electronic states as presented e.g. in Figure 1 of ref. [17]. Autoionization and dissociation of the $\mathrm{Q}_{1}$ or $\mathrm{Q}_{2}$ states occur on a comparable time scale of few femtoseconds, in conditions where the electronic dynamics is identical for the two isotopes, although their nuclear motion characteristic time is different. Comparing DPI for $\mathrm{H}_{2}$ and $\mathrm{D}_{2}$, at the level of the most sensitive observables describing electron-ion kinetic energy correlation and molecular 
frame photoemission induced by circularly polarized light, gives access to original information about the coupling between the electron and nuclei motion which mediates the DPI reaction. These results motivate future projects where PI will be induced by femtosecond VUV pulses produced by a $1 \mathrm{kHz}$ high order harmonic generation source. In the second part we illustrate the extension of the VC method using a light source of $1 \mathrm{kHz}$ repetition rate by results obtained for multiphoton PI of the Xe atom induced by $70 \mathrm{fs}$ pulses centered at $400 \mathrm{~nm}$.

\section{EXPERIMENTAL METHOD}

The double velocity spectrometer [27] (Figure 1), equipped with two delay-line position sensitive detectors (PSDs: DLD40 Roentdek) [28], combines time-of-flight-resolved ion-electron coincidence detection and imaging techniques. The interaction region is located at the center of the spectrometer and defined at the intersection of the supersonic molecular jet and the light beam.

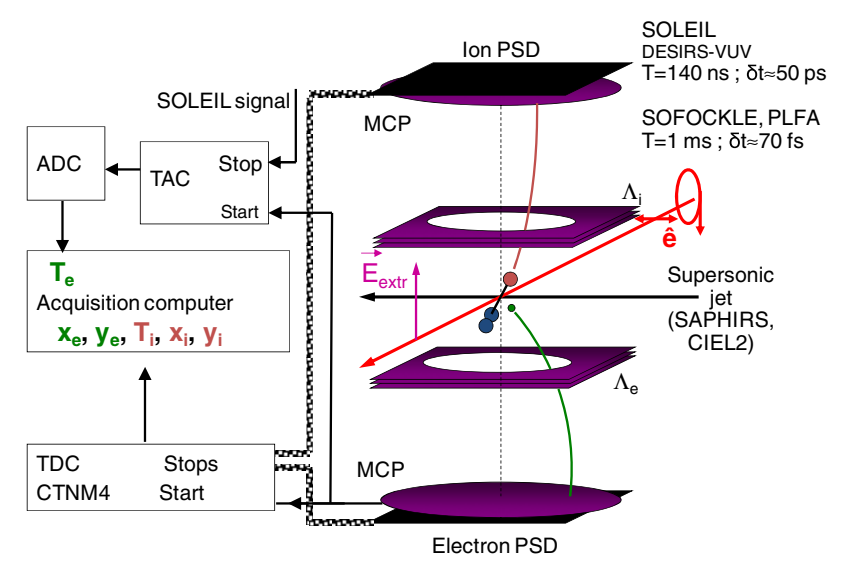

Figure 1. Scheme of the electron-ion velocity spectrometer and the acquisition set-up (see text).

Ions and electrons are extracted from the interaction region by a DC uniform electric field and guided to their respective PSD through an intermediate region where two focusing electrostatic lens sets $\left(\Lambda_{\mathrm{A}+}\right.$ and $\left.\Lambda_{\mathrm{e}}\right)$ are applied. The magnitude of the extraction field, of the order of $150 \mathrm{~V} / \mathrm{cm}$ for the study of $\mathrm{H}_{2} / \mathrm{D}_{2} \mathrm{DPI}$, is chosen such that the spectrometer ensures a $4 \pi$ collection of the electrons and ion fragments. For each $\left(\mathrm{A}^{+}, \mathrm{e}_{\mathrm{ph}}\right)$ coincident event the three components of the $\mathbf{V}_{\mathrm{A}+}$ ion fragment and $\mathbf{V}_{\mathrm{e}}$ photoelectron emission velocity vectors are deduced from the time of flight (TOF) and impact position of the particles on the PSDs.

The signal detected on the front multi-channel plate of the electron detector is used as a common start for a 8 independent channel time-to-digital converter (CTN-TDC [29], encoding resolution 250 ps) and a time-to-amplitude converter (TAC). The TAC is stopped by the electronic signal synchronous with the light pulse, providing the electron time of flight $\left(\mathrm{TOF}_{\text {elec }}\right)$ with a coding resolution of $25 \mathrm{ps}$ on the $100 \mathrm{~ns}$ scale. Four channels of the CTN-TDC are dedicated to the time signals from the ends of the two delay lines of the electron detector leading to the electron position, and the four others to those of the ion detector providing the ion position and time of flight $\left(\mathrm{TOF}_{\text {ion }}\right)$ [27]. Typical values of $\mathrm{TOF}_{\text {elec }}$ and $\mathrm{TOF}_{\text {ion }}$ are in the range of $10-50 \mathrm{~ns}$ and few $\mu$ s, respectively.

The $\mathrm{H}_{2} / \mathrm{D}_{2}$ DPI experiments have been performed at the synchrotron radiation SOLEIL operated in the eight-bunch mode (period $\mathrm{T}=140 \mathrm{~ns}$, pulse width $\delta \mathrm{t}=50 \mathrm{ps}$ ), on the undulator-based VUV beamline DESIRS [30] delivering circularly or linearly polarized light, using the supersonic expansion of the SAPHIRS set-up [31]. The reported results of multiphoton PI of Xe rely on two series of experiments using $70 \mathrm{fs}$ pulses centered at $400 \mathrm{~nm}$ delivered by the SOFOCKLE and PLFA sources 
(SLIC, CEA-Saclay) after frequency doubling of a $1 \mathrm{kHz}$ Ti:sapphire laser. We compared there the experimental conditions provided by using the supersonic SAPHIRS expansion on one side, and that of the ultrahigh vacuum set-up CIEL2 [32] where the molecular expansion is defined by a two skimmer device. The count rate conditions for the study of PI based on the coincidence VC method, involving either the synchrotron radiation or the femtosecond laser light sources, run at a frequency of $7 \mathrm{MHz}$ and $1 \mathrm{kHz}$, respectively, are briefly discussed in section 4.

\section{DISSOCIATIVE PHOTOIONIZATION OF $\mathrm{H}_{2}$ AND $\mathrm{D}_{2}$ INDUCED BY CIRCULARLY POLARIZED SYNCHROTRON RADIATION AT $h v=32.5 \mathrm{eV}$}

\subsection{DPI of $\mathrm{H}_{2}$ and $\mathrm{D}_{2}$ : Electron-ion kinetic energy correlation, ion fragment asymmetry parameters}

The $\left(\mathrm{E}_{\mathrm{H}+}, \mathrm{E}_{\mathrm{e}}\right)$ (a) and $\left(\mathrm{E}_{\mathrm{D}+}, \mathrm{E}_{\mathrm{e}}\right)$ kinetic energy correlation diagrams (KECDs), i.e. the bidimensional histogram of the $\left(\mathrm{H}^{+} / \mathrm{D}^{+}\right.$, e) coincident events, derived from the analysis of the magnitude of the $\left(\mathbf{V}_{\mathrm{H} / \mathrm{D}+}, \mathbf{V}_{\mathrm{e}}\right)$ vectors independent on the polarization of the light, are displayed in Figure 2 for $h v=$ $32.5 \mathrm{eV}$. The $\mathrm{L}_{1}$ ground state dissociation limit $\left(\mathrm{H}^{+} / \mathrm{D}^{+}+\mathrm{H} / \mathrm{D}(1 \mathrm{~s})\right)$ which lies at $\varepsilon_{\mathrm{D}}=18.076 \mathrm{eV}$ above the $\mathrm{H}_{2}\left(\mathrm{X}^{1} \Sigma_{\mathrm{g}}^{+}, \mathrm{v}=0\right)$ reference, fixed as the origin in potential energy, is the only DPI channel opened: it is represented on the KECD as a diagonal line of slope -2 .
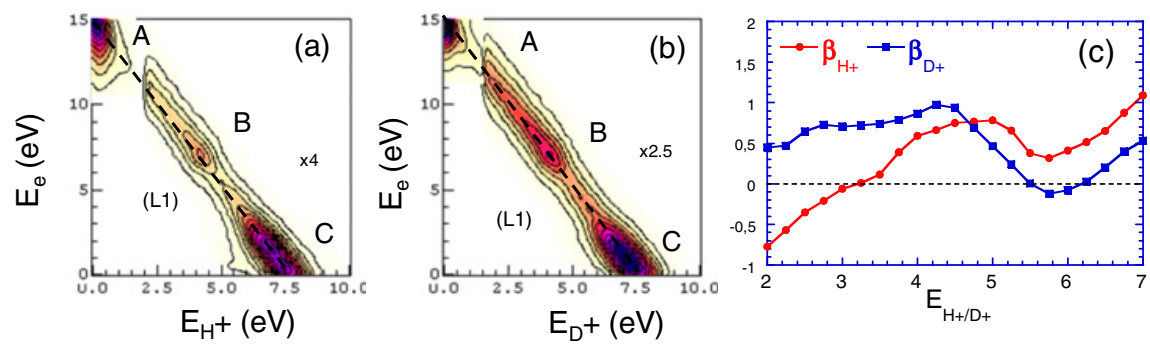

Figure 2. $\left(\mathrm{E}_{\mathrm{H}+}, \mathrm{E}_{\mathrm{e}}\right)(\mathrm{a})$ and $\left(\mathrm{E}_{\mathrm{D}+}, \mathrm{E}_{\mathrm{e}}\right)$ (b) KECDs for $h v=32.5 \mathrm{eV}$, extraction field $150 \mathrm{~V} / \mathrm{cm}$, intensity scale runs from white (lowest intensity) to black (highest intensity) in linear scale with contour lines spaced by $10 \%$ of the maximum value; (c) Evolution of the asymmetry parameters (the error bars not shown are \pm 0.05 ).

The two KECDs display similarities. Apart from peak A, which corresponds to ionization into the dissociative continuum of the $\mathrm{H}_{2}^{+}\left(\mathrm{X}^{2} \Sigma_{\mathrm{g}}^{+}\right)$ground state taking place in the FC region, the KECDs show a continuous distribution of the DPI events along $\mathrm{L}_{1}$, with two broad maxima, labelled $\mathrm{B}$ and $\mathrm{C}$, (B: $\left.\mathrm{E}_{\mathrm{e}} \approx 7 \mathrm{eV}, \mathrm{E}_{\mathrm{H} / \mathrm{D}+} \approx 4 \mathrm{eV}\right)$ and $\left(\mathrm{C}: \mathrm{E}_{\mathrm{e}} \approx 1.2 \mathrm{eV}, \mathrm{E}_{\mathrm{H} / \mathrm{D}+} \approx 6.6 \mathrm{eV}\right)$, consistent with the kinetic energy distributions (KEDs) reported previously for these two processes [33,34]. These structures have been assigned to autoionization of selected $\mathrm{Q}_{1}$ and $\mathrm{Q}_{2}$ resonant states taking place outside the FC region, during dissociation [35]. The relative importance of peak $\mathrm{B}$ is larger for $\mathrm{D}_{2}$ than for $\mathrm{H}_{2}$. Furthermore the evolution of the $\beta_{\mathrm{H}+}$ and $\beta_{\mathrm{D}+}$ asymmetry parameters, which characterizes the ion fragment angular distribution with respect to the linear polarization axis, as a function of the proton energy displays significant differences as shown in Figure 2(c). This result illustrates clearly that the relative contribution of autoionization from states of $\Sigma(\Delta \Lambda=0, \beta=2)$ and $\Pi(\Delta \Lambda= \pm 1, \beta=-1)$ symmetries depends on the internuclear distance in a different way for PI of $\mathrm{H}_{2}$ and $\mathrm{D}_{2}$. It confirms the conclusions obtained from the analysis of the KED of the ions fragments [34] at such photon energies. The dominant reactions can be summarized as follows: although direct ionization into the $\mathrm{H}_{2}^{+}\left({ }^{2} \Sigma_{\mathrm{u}}^{+}\right)$ionic state selectively

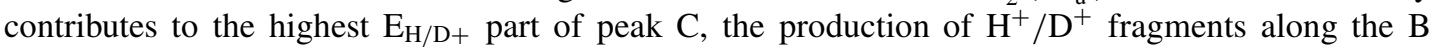
and $\mathrm{C}$ structures is governed by the contribution of autoionization of $\mathrm{Q}_{1}$ and $\mathrm{Q}_{2}$ resonant states into the $\mathrm{H}_{2}^{+}\left(\mathrm{X}^{2} \Sigma_{\mathrm{g}}^{+}\right)$(dissociative continuum) and $\mathrm{H}_{2}^{+}\left({ }^{2} \Sigma_{\mathrm{u}}^{+}, 2 \mathrm{p} \sigma_{\mathrm{u}}\right)$ (repulsive) ionic states. Autoionization 
(AI) of the lowest $\mathrm{Q}_{2}\left({ }^{1} \Pi_{\mathrm{u}}(1)\right)$ state of the $\mathrm{Q}_{2}$ series gives two main contributions to the perpendicular transition with the maxima observed for peaks B and C: they involve AI into the $\left(\mathrm{X}^{2} \Sigma_{\mathrm{g}}^{+}\right)$and $\left({ }^{2} \Sigma_{\mathrm{u}}^{+}\right)$ ionic states [35], respectively, in a comparable way for the $\mathrm{H}_{2}$ and $\mathrm{D}_{2}$ isotopes. AI of $\mathrm{Q}_{1}\left({ }^{1} \Sigma_{\mathrm{u}}^{+}\right)$states into the $\left(\mathrm{X}^{2} \Sigma_{\mathrm{g}}^{+}\right)$ionic ground state demonstrates a significant isotope effect most visible in the parallel component of the transition: it mainly contributes to peak B for DPI of the $\mathrm{D}_{2}$ molecule whereas it is shifted to higher proton energies for DPI of the $\mathrm{H}_{2}$ molecule, a fingerprint that AI of these states occurs at larger internuclear distance for $\mathrm{H}_{2}$ than for $\mathrm{D}_{2}$. These contrasted observations must take into account the different lifetimes of the relevant autoionizing states which mediate the dynamics of DPI, and their comparison with the characteristic dissociation time of the molecule, shorter for $\mathrm{H}_{2}$ than for $\mathrm{D}_{2}$.

\subsection{DPI of $\mathrm{H}_{2}$ and $\mathrm{D}_{2}$ : Molecular frame photoemission}

In order to derive the MFPADs for selected processes in the KECDs, we use the general formalism developed earlier in the dipole approximation for one-photon photoionization of a linear molecule induced by linearly [18] or circularly [3] polarized light. In the general expression of the $I\left(\chi, \theta_{e}, \phi_{e}\right)$ MFPAD, all the dynamical information is contained in five one-dimensional $F_{L N}\left(\theta_{e}\right)$ functions, the dependence in terms of the molecular axis orientation $\chi$ with respect to the light propagation axis $\mathbf{k}$ and the photoelectron azimuthal emission angle in the molecular frame $\phi_{e}$ being expressed by simple geometrical functions (see ref. $[3,12,18]$ for the analytical expansions and the extraction of the $F_{L N}\left(\theta_{e}\right)$ functions). The expansion of the $F_{L N}\left(\theta_{e}\right)$ functions in terms of Legendre polynomials gives access to the partial wave dependent complex dipole matrix elements, their magnitude and relative phase. The data can also be conveniently presented as shown in Figure 3 in terms of selected MFPADs for any orientation of the molecular axis with respect to linearly or circularly polarized light once the $F_{L N}\left(\theta_{e}\right)$ functions are determined, using the expression of $I\left(\chi, \theta_{e}, \phi_{e}\right)$, as e.g.:

$$
\begin{aligned}
I_{\chi=0}\left(\theta_{e}\right) & =F_{00}\left(\theta_{e}\right)+F_{20}\left(\theta_{e}\right) \\
I_{\chi=90}\left(\theta_{e}, \phi_{e}\right) & =F_{00}\left(\theta_{e}\right)-0.5 F_{20}\left(\theta_{e}\right)+3 F_{22}\left(\theta_{e}\right) \cos \left(2 \phi_{e}\right) \\
I_{+1 \chi=90}\left(\theta_{e}, \phi_{e}\right) & =F_{00}\left(\theta_{e}\right)+0.25 F_{20}\left(\theta_{e}\right)+F_{11}\left(\theta_{e}\right) \sin \left(\phi_{e}\right)-1.5 F_{22}\left(\theta_{e}\right) \cos \left(2 \phi_{e}\right)
\end{aligned}
$$
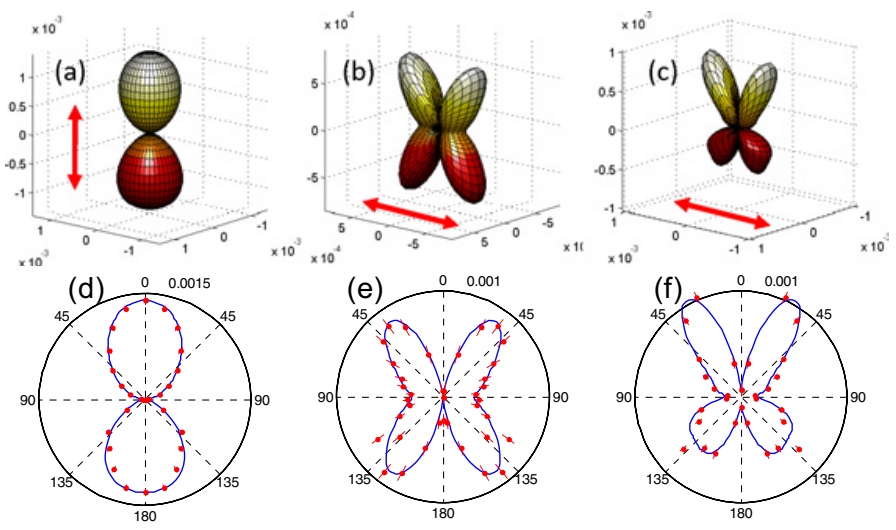

Figure 3. MFPADs for $\mathrm{E}_{\mathrm{H} / \mathrm{D}+} \approx 3 \mathrm{eV}$ (band-width $0.5 \mathrm{eV}$ ) (a) DPI of $\mathrm{D}_{2}: I\left(\theta_{e}, \phi_{\mathrm{e}}\right)$ MFPAD for a molecular axis parallel to linearly polarized light $\mathbf{P}\left(\chi=0^{\circ}\right)$ (b) DPI of $\mathrm{H}_{2}$ : MFPAD for a molecular axis perpendicular to $\mathbf{P}$ $\left(\chi=90^{\circ}\right)(\mathrm{c}) \mathrm{DPI}$ of $\mathrm{D}_{2}$ for $\mathrm{E}_{\mathrm{H} / \mathrm{D}+} \approx 4 \mathrm{eV}$ : MFPAD for a molecular axis perpendicular to $\mathbf{P}\left(\chi=90^{\circ}\right)$. (d-f) cuts of the MFPADs $(\mathrm{a}-\mathrm{c})$ in the plane defined by the molecular and the polarization axes. 
Figure 3 displays three examples of MFPADs: for $\mathrm{E}_{\mathrm{H} / \mathrm{D}+} \approx 3 \mathrm{eV}$, where the isotope effect is large in terms of asymmetry parameter, we present the MFPADs for DPI of $\mathrm{D}_{2}\left(\chi=0^{\circ}\right.$ : dominant parallel transition) and $\mathrm{H}_{2}\left(\chi=90^{\circ}\right.$ : dominant perpendicular transition). The MFPAD (a) reveals the dominant contribution of a $\mathrm{p} \sigma_{\mathrm{u}}$ partial wave, consistent with ionization from a $\mathrm{Q}_{1}\left({ }^{1} \Sigma_{\mathrm{u}}^{+}\right)$state into the $\left(\mathrm{X}^{2} \Sigma_{\mathrm{g}}^{+}\right)$state, whereas the MFPAD (b) may be assigned either to a $\mathrm{d} \pi_{\mathrm{g}}$ dominant partial wave, or to a superposition of $\mathrm{p} \pi_{\mathrm{u}}$ and $\mathrm{f} \pi_{\mathrm{u}}$ waves. The latter attribution fits the previous assignment of AI of the $\mathrm{Q}_{2}\left({ }^{1} \Pi_{\mathrm{u}}(1)\right)$ resonant state into the $\left(\mathrm{X}^{2} \Sigma_{\mathrm{g}}^{+}\right)$state in this energy region [34,35]. The MFPAD (c) for DPI of $\mathrm{D}_{2}$ about $\mathrm{E}_{\mathrm{H} / \mathrm{D}+} \approx 4 \mathrm{eV}$ displays a striking forward-backward (FW-BW) asymmetry [25], also observed for $\mathrm{H}_{2}$ for the same energy range [17]. Such emission anisotropies have been attributed to the interference between the two channels involving autoionization of the $\mathrm{D}_{2}\left(\mathrm{Q}_{2}\left({ }^{1} \Pi_{\mathrm{u}}\right)\right)$ state into the $\mathrm{H}_{2}^{+} / \mathrm{D}_{2}^{+}\left(\mathrm{X}^{2} \Sigma_{\mathrm{g}}^{+}\right)$

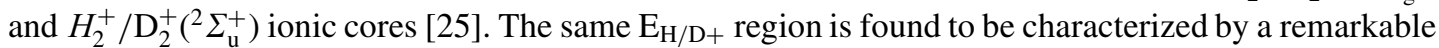
circular dichroism effect as observed in preliminary measurements for $\mathrm{D}_{2}$ [26] rather comparable for the two isotopes, which will be discussed in another publication.

\section{MULTIPHOTON PHOTOIONIZATION OF XE INDUCED BY FEMTOSECOND LASER PULSES}

In this section we briefly report results of a multiphoton PI reaction study using the VC method. Although electron-ion coincidence experiments have become possible thanks to the $\mathrm{kHz}$ repetition rate of the femtosecond lasers delivering visible or UV photons, this frequency remains rather low compared to the $\mathrm{MHz}$ range of the synchrotron radiation (SR) sources. Indeed, performing coincidence measurements requires that a maximum of one event occurs per pulse, which imposes that in average the count rate must be kept such that much less than one event occurs per pulse. Whereas this condition is satisfied and leads to appreciable acquisition statistics using SR, the count rates must be kept rather low when one turns to a $\mathrm{kHz}$ source. For a detailed discussion we refer to previous studies [36-38] and a forthcoming paper [39] and we illustrate below the conditions for the present report. Figure 4 displays a section of the time of flight (TOF) spectrum of the $\mathrm{Xe}^{+}$ions produced by 4-photon ionization of $\mathrm{Xe}$ using $398 \mathrm{~nm}-70 \mathrm{fs}$ photons delivered by the femtosecond laser source PLFA (SLIC Saclay) obtained using the CIEL 2 ultrahigh vacuum supersonic expansion.
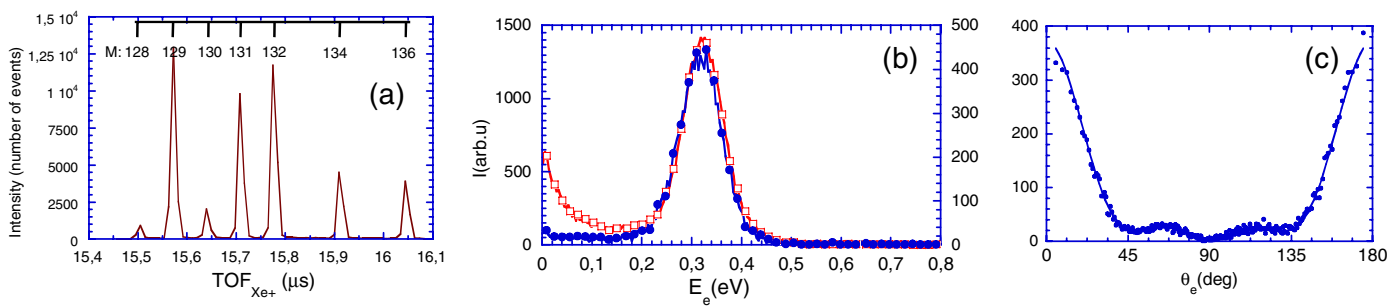

Figure 4. (a) $\mathrm{Xe}^{+}$TOF spectrum, extraction field $20 \mathrm{~V} / \mathrm{cm}$ (b) photoelectron energy spectra for selection of a single isotope: filled and empty labels are for an experiment performed with $40 \mathrm{c} / \mathrm{s}$ and $170 \mathrm{c} / \mathrm{s}$, respectively (c) photoelectron angular distribution with respect to the linear polarization axis.

The well resolved $\mathrm{Xe}^{+}$TOF spectrum enables mass selection of one isotope, for which the photoelectron spectra are reported in two different acquisition conditions. The peak at $0.32 \mathrm{eV}$ characterizes PI into the $\mathrm{Xe}^{+}\left({ }^{2} \mathrm{P}_{3 / 2}\right)$ ground state after absorption of four photons at $398 \mathrm{~nm}$. For the highest rate of $170 \mathrm{c} / \mathrm{s}$ (coincidences per second) a tail for low electron energies is observed: it corresponds to the contribution of false coincidences between the selected $\mathrm{Xe}^{+}$ion and an electron produced by another mass (contribution of ionized residual gas) and it disappears when the acquisition rate is reduced to $40 \mathrm{c} / \mathrm{s}$. A detailed study taking into account the electron and ion count rates and the detector's 
efficiency shows that an acquisition rate $20-50 \mathrm{c} / \mathrm{s}$ ensures real coincidence conditions [39], i.e. a maximum value of the order of 0.05 coincidence per pulse in average, comparable to the conditions in [38]. Figure 4(c) displays the photoelectron angular distribution which is strongly aligned along the linear polarization axis and reveals several oscillations. When analyzed using a Legendre polynomial expansion, according to the expression $I\left(\theta_{e}\right) \propto \Sigma_{k=0}^{2 N} \beta^{(k)} P_{k} \cos \left(\theta_{e}\right)$, where $\mathrm{N}$ is the number of absorbed photons, three asymmetry parameters $\beta_{e}$ of different orders are needed to describe the distribution $\beta_{e}^{(2)} \approx 2.6 \pm 0.1, \beta_{e}^{(4)} \approx 2.4 \pm 0.1$ and $\beta_{e}^{(6)} \approx 1.7 \pm 0.1$. These values imply that ionization involves the absorption of four photons [40]. The $\mathrm{VC}$ method has been applied in comparable conditions for the study of multiphoton DPI of the $\mathrm{NO}_{2}$ and $\mathrm{CF}_{3} \mathrm{I}$ molecules.

\section{CONCLUSION}

In this contribution we have presented the observables achieved by using the vector correlation method for the study of photoionization of small molecules. We have reported selected results of a comparative study of PI of the $\mathrm{H}_{2}$ and $\mathrm{D}_{2}$ molecules by VUV synchrotron radiation circularly polarized light involving the resonant excitation of $\mathrm{Q}_{1}\left(2 \mathrm{p} \sigma_{\mathrm{u}}, \mathrm{nl} \lambda\right)$ and $\mathrm{Q}_{2}\left(2 \mathrm{p} \pi_{\mathrm{u}}, \mathrm{nl} \lambda\right)$ doubly excited states. The detailed analysis of the electron-ion kinetic energy correlation and the ion fragment asymmetry parameters demonstrate significant isotope effects attributed to the coupling of electron and nuclei motion in DPI of $\mathrm{H}_{2}$ and $\mathrm{D}_{2}$ involving excitation of the $\mathrm{Q}_{1}\left({ }^{1} \Sigma_{\mathrm{u}}^{+}\right)$state. Determination of the MFPADs for a molecule aligned parallel or perpendicular to the polarization axis of linearly polarized light enables the identification of the dominant partial waves characterizing the electronic wave function in the continuum. Their significant variation with the $\mathrm{E}_{\mathrm{H} / \mathrm{D}+}$ kinetic energy reveals a dependence of the PI outcome as a function of the internuclear distance when dissociation occurs and it characterizes the existence of interference phenomena between undistinguishable reaction pathways. A more detailed discussion of the experimental results and their comparison with four-body calculations, involving notably the circular dichroism effects which characterize molecular frame photoemission, will be reported in a forthcoming paper. The VC method has recently been extended to the study of multiphoton $\mathrm{PI}$ induced by $\mathrm{kHz}$ femtosecond lasers as demonstrated by the report of results for PI of the Xe atom induced by absorption of four $400 \mathrm{~nm}$ photons.

These results motivate future projects where PI will be induced by femtosecond VUV pulses produced by a $1 \mathrm{kHz}$ high order harmonic generation source in the Attophysics group (SPAM, Saclay). Dynamical effects in one-photon and two-photon resonant PI of the $\mathrm{H}_{2}$ molecule evidenced by ab-initio four body calculations have been reported [41]. Such effects will be investigated at the level of the energy and angular observables provided by the VC method.

\section{Acknowledgements}

We gratefully acknowledge the support of S. Damoy, engineer at the LCAM. We are very grateful to A. Huetz and E. Bouisset (LIXAM) for their collaboration and help in running the CIEL2 set-up. We gratefully acknowledge L. Nahon and G. Garcia, beamline scientists for DESIRS at SOLEIL, J.F. Gil engineer, and the machine department staff for operating SOLEIL, as well as C. Cornaggia (SOFOCKLE) and O. Gobert, D. Garzella, P. Breger, M. Perdrix, JF Hergott (PLFA) at the SLIC laser source facility (SPAM, CEA Saclay).

\section{References}

[1] D. Dill, J. Chem. Phys. 65, (1976) 1130; J.W. Davenport, Phys. Rev. Lett. 36, (1976) 945

[2] R. R. Lucchese, J. Electron. Spectro. Relat. Phenom. 141, (2004) 201

[3] K. L. Reid, Ann. Rev. Phys. Chem. 54, (2003) 397

[4] M. Lebech, A. Lafosse, J. C. Houver, D. Dowek, C. Alcaraz, L. Nahon, R.R. Lucchese J. Chem. Phys., 118, (2003) 9653 
[5] F. Martin, J. Phys. B 32, (1999) R197 and ref. therein.

[6] M. Lebech, J.C. Houver, D. Dowek and R.R. Lucchese, Phys. Rev. Lett. 96, (2006) 073001

[7] N.A. Cherepkov, G. Raseev, J. Adachi, Y. Hikosaka, K. Ito, S. Motoki, M. Sano, K. Soejima and A. Yagishita, J. Phys. B 33, (2000) 4213

[8] O. Gessner, Y. Hikosaka, B. Zimmermann, A. Hempelmann, R.R. Lucchese, J.H.D. Eland, P.M. Guyon, U. Becker, Phys. Rev. Lett. 88, (2002) 193002

[9] H. Stapelfeldt and T. Seideman, Rev. Mod. Phys., 75, (2003) 543-557

[10] I. Thomann, R. Lock, V. Sharma, E. Gagnon, S. T. Pratt, H. C. Kapteyn, M. M. Murnane and W. Li, J. Phys. Chem. A, 112, (2008) 9382

[11] A. Lafosse, M. Lebech, J. C. Brenot, P. M. Guyon, O. Jagutzki, L. Spielberger, M. Vervloet, J. C. Houver, and D. Dowek, Phys. Rev. Lett. 84, 5987 (2000)

[12] D. Dowek, in J. Ullrich and V.P. Shevelko (Eds.): Many-Particle Quantum Dynamics in Atomic and Molecular Fragmentation (Springer, Berlin-Heidelberg 2003).

[13] R. Dörner et al, Phys. Rep. 330, (2000) 95; J. Ullrich et al, Rep. Prog. Phys. 66, 1463 (2003).

[14] A.V. Golovin, N.A. Cherepkov and V.V. Kuznetsov, Z. Phys. D 24, (1992) 371

[15] P. Downie, I. Powis, Phys. Rev. Lett. 82, (1999) 2864

[16] Y. Hikosaka and J.H.D. Eland, J. Phys. B 33, (2000) 3137

[17] A. Lafosse , M. Lebech, J.C. Brenot, P.M. Guyon, L.Spielberger, O. Jagutzki, J.C. Houver and D. Dowek, J. Phys. B: At . Mol. Phys. 36, (2003) 4683

[18] R. R. Lucchese, A. Lafosse, J. C. Brenot, P. M. Guyon, J. C. Houver, M. Lebech, G. Raseev, and D. Dowek, Phys. Rev. A 65, (2002) 020702

[19] M. Lebech, J.C. Houver, R.R. Lucchese and D. Dowek, J. Chem. Phys. 117, (2002) 9248; A. Lafosse, J.C. Brenot, P.M. Guyon, J.C. Houver, A.V. Golovin, M. Lebech and D. Dowek, P. Lin and R.R. Lucchese, J. Chem. Phys. 117, (2002) 8368

[20] W.B. Li, R. Montuoro, J.C. Houver, A. Haouas, L. Journel, M. Simon, R.R. Lucchese, and D. Dowek, Phys. Rev. A 75, (2007) 052718; W.B. Li et al J. Electron. Spectro. Relat. Phenom., 156-158, (2007) 30; L. Journel et al, Phys. Rev. A, 77042710 (2008).

[21] F. Heiser, O. Gessner, J. Viefhaus, K. Wieliczek, R. Hentges, and U. Becker, Phys. Rev. Lett. 79, (1997) 2435

[22] A. Landers et al Phys. Rev. Lett. 87, (2001) 013002; T. Jahnke et al Phys. Rev. Lett. 88, (2002) 073002 ; M. Schöffler et al, Science 320, (2008)892

[23] N. Saito et al J. Phys. B 36, (2003) L25, J. Phys. B. 38, (2005) L277; H. Fukuzawa et al, J. Phys. B 41, (2008) 045102.

[24] R.R. Lucchese, R. Carey, C. Elkharrat, J.C. Houver, D. Dowek, J. Phys.: Conf. Ser. 141, (2008) 012009

[25] F. Martin et al, Science 315, (2007) 629

[26] D. Dowek, J. Fernandez, M. Lebech, J.C. Houver and F. Martin, J. Phys.: Conf. Ser. 88, (2007) 012009

[27] M. Lebech, J.C. Houver and D. Dowek, Rev. Sci. Instrum. 73, (2002) 1866

[28] Roentdek-Handels GmbH, Kelkheim, Germany.

[29] IPNO "Détection: Temps, Position, Image" (DTPI) Technology Division (supported by the MRCT-CNRS, Federation FR2764 and by the Université Paris-Sud)

[30] L. Nahon, C. Alcaraz, J.-L. Marlats, B. Lagarde, F. Polack, R. Thissen, D. Lepère, and K. Ito, Rev. Sci. Instrum. 72, (2001) 1320; L. Nahon and C. Alcaraz, Applied Optics, 43, (2004) 1024

[31] M. Richard-Viard, A. Delboulbé, and M. Vervloet, Chem. Phys. 209, (1996) 159

[32] O. Guyétand, M. Gisselbrecht, A. Huetz, P. Agostini, B. Carré, P. Breger, O. Gobert, D. Garzella, J-F Hergott, O Tcherbakoff, H. Merdji, M. Bougeard, H. Rottke, M. Böttcher, Z. Ansari, P. Antoine and L F DiMauro, J. Phys. B 41, (2008) 065601

[33] S. Strathdee and R. Browning, J. Phys. B: At . Mol. Phys. 12, (1979) 1789; C.J. Latimer, J. Geddes, M.A. MacDonald, N. Kouchi and K.F. Dunn, J. Phys. B: At . Mol. Phys. 29, (1996) 
6113.

[34] K. Ito, R. Hall and M. Ukai, J. Chem. Phys. 104, (1996) 8449

[35] I. Sanchez and F. Martin, Phys. Rev. Lett. 79, (1997) 1654, Phys. Rev. Lett. 82, 3775 (1999)

[36] V. Stert, W. Radloff, Th. Freudenberg, F. Noack, I.V. Hertel, C. Jouvet, C. Dedonder-Lardeux and D. Solgadi, Europhys. Lett. 40, (1997) 515; V. Stert, W. Radloff, C.P. Schultz and I.V. Hertel, Eur. Phys. J. D. 5, (1999) 97

[37] J.A. Davies, R.E. Continetti, D.W. Chandler, and C.C. Hayden, Phys. Rev. Lett. 84, 5983 (2000)

[38] A. Vredenborg, W.G. Roeterdink, and M.H.M. Janssen, J. Chem. Phys. 128, (2008) 204311

[39] C. Elkharrat et al, in preparation

[40] S.J. Bajic, R.N. Compton, X. Tang, P. Lambropoulos, Phys. Rev. A 44, (1991) 2102 ; C. Bordas et al Phys. Rev. A, 51, (1995) 3726

[41] J. L. Sanz-Vicario, H. Bachau, F. Martín, PRA 73, (2006) 033410 ; A. Palacios, H. Bachau, F. Martin, Phys. Rev. Lett. 96, (2006) 143001, Phys. Rev. A 75, (2007) 013408 